Growth and properties of III-V compound semiconductor heterostructure nanowires

This article has been downloaded from IOPscience. Please scroll down to see the full text article.

2011 Semicond. Sci. Technol. 26014035

(http://iopscience.iop.org/0268-1242/26/1/014035)

View the table of contents for this issue, or go to the journal homepage for more

Download details:

IP Address: 130.56.107.19

The article was downloaded on 24/06/2011 at 02:24

Please note that terms and conditions apply. 


\title{
Growth and properties of III-V compound semiconductor heterostructure nanowires
}

\author{
Q Gao ${ }^{1}$, H H Tan ${ }^{1}$, H E Jackson ${ }^{2}$, L M Smith ${ }^{2}$, J M Yarrison-Rice ${ }^{3}$, \\ Jin Zou ${ }^{4}$ and C Jagadish ${ }^{1}$ \\ ${ }^{1}$ Department of Electronic Materials Engineering, Research School of Physics and Engineering, The \\ Australian National University, Canberra, ACT 0200, Australia \\ ${ }^{2}$ Department of Physics, University of Cincinnati, Cincinnati, OH 45221-0011, USA \\ ${ }^{3}$ Department of Physics, Miami University, Oxford, OH 45056, USA \\ ${ }^{4}$ School of Engineering and Centre for Microscopy and Microanalysis, The University of Queensland, \\ QLD 4072, Australia
}

E-mail: qiang.gao@anu.edu.au and chennupati.jagadish@anu.edu.au

Received 18 June 2010, in final form 2 September 2010

Published 15 December 2010

Online at stacks.iop.org/SST/26/014035

\begin{abstract}
We review various GaAs-based axial and radial nanowire heterostructures grown on (1 111 ) B GaAs substrates by metal organic chemical vapor deposition via vapor-liquid-solid mechanism. Transmission electron microscopy, time-resolved photoluminescence and micro-Raman spectroscopy have been used to understand the crystal structure, non-radiative surface and bulk defects, carrier lifetime and strain effects on the bandgap energy.
\end{abstract}

(Some figures in this article are in colour only in the electronic version)

\section{Introduction}

Semiconductor devices and modern solid-state physics have advanced significantly since the double heterostructure (DHS) laser was proposed independently by Alferov and Kazarinov [1], and Kroemer [2]. DHS has been a revolutionary idea to more precisely control the fundamental parameters in semiconductor crystals and devices, such as the bandgap energy, the effective mass and mobility of charge carriers and the refractive index. One cannot imagine our everyday lives without semiconductor DHS devices ranging from DHS lasers, to light-emitting diodes, bipolar transistors and lownoise, high-electron-mobility transistors.

In the last decade, nanowire heterostructures have come into focus in the field of semiconductor nanomaterials. These quasi-one dimensional structures offer unique opportunities to realize novel nanoscale semiconductor devices. III$\mathrm{V}$ compound semiconductor nanowires are of particular interest for optoelectronic devices due to their high optical emission efficiency compared to indirect-bandgap group IV crystals. Various III-V compound semiconductor nanowirebased devices, including solar cells [3], single photon emitters
[4-6], photodetectors [7, 8], biosensors [9], light emitting diodes [5, 10], lasers [11-13] and single electron devices [14], have already been demonstrated. Unlike traditional epitaxy which is based on layer-by-layer deposition on a planar surface, nanowires grow as free-standing structures on a wafer offering the flexibility to construct a true three-dimensional architecture which is not possible otherwise. The typical nanowire is grown from a seed metal particle, the size of which also determines the diameter of nanowires. The growth mechanism is often described as a vapor-liquid-solid (VLS) mechanism, first proposed by Wagner and Ellis for silicon whiskers grown with a Au particle [15], although a vaporsolid-solid growth is also possible in some material systems $[16,17]$. In the VLS growth, the liquid metal droplet becomes a sink for reaction species supplied from the surrounding vapor, and is readily supersaturated with reaction species. The precipitation of reaction species at the solid/liquid interface leads to highly anisotropic nanowire growth. This growth mechanism has been extremely flexible for heterostructures to form in both axial and radial directions. For example, nanowire heterostructures can be grown in either the axial direction by alternating precursors or the radial direction 
(core-shell) by increasing growth temperature. This flexibility provides a rich environment for designing and fabricating novel nanostructures for illuminating new physics or enabling new technologies. On the other hand, due to the large nanowire surface area in comparison to its volume and the small contact area between a nanowire and its substrate, Zervos et al have shown theoretically that the strain induced at heterointerfaces in nanowires can be elastically relieved over a thickness of a few monolayers [18]. Experimentally, the formation of defectfree and sharp heterojunctions has been demonstrated in highly lattice mismatched $\mathrm{GaAs} / \mathrm{GaP}$, InAs/InP, GaAs/GaSb and InAs/InSb nanowires [19-23]. However, challenges always accompany these new opportunities. For example, in nanowire heterostructures, random kinking or branching imposes a great challenge in their growth [24, 25]. Precise control over the kinking location and direction is crucial to grow sophisticated 3D nanowire structures.

III-V semiconductor, in particular, GaAs-based heterostructure nanowires are promising for optoelectronic devices due to the superior electrical and optical properties over other material systems. The GaAs material system has already demonstrated their great importance in the current semiconductor industries for optoelectronic and electronic devices, for example, the GaAs/AlGaAs quantum well and InAs/GaAs quantum dot lasers and photodetectors. Accordingly, GaAs-based heterostructure nanowires are prime candidates for electrically and optically active nanowire devices [3, 4, 11]. Since there were two good review papers covering synthesis techniques and physical properties of heterostructure nanowires [26, 27], we hereby only focus on a few GaAs-based nanowire heterostructures synthesized by our group to understand the growth control and their implications on optical properties. First, we briefly review the experimental methods for nanowire growth, structural and optical characterization. Then, we describe the current advances and characteristic properties of several specific heterostructures including GaAs/AlGaAs, GaAs/GaP coreshell and GaAs-InAs, GaAs-GaSb axial heterostructures.

\section{Growth and characterization technique}

All nanowires were grown on GaAs (1 111 ) B substrates except $\mathrm{GaAs} / \mathrm{GaP}$ nanowires which were grown on $\mathrm{Si}$ (1 111 ) (4 $4^{\circ}$ miscut) substrates using a horizontal flow metal organic chemical vapor deposition (MOCVD) reactor at a pressure of 100 mbar with ultrahigh purity hydrogen as the carrier gas. Trimethylgallium (TMG), trimethylaluminum (TMA) and trimethylindium (TMI) precursors were used for group III and $\mathrm{AsH}_{3}, \mathrm{PH}_{3}$ and trimethylantimony (TMSb) precursors were used for group $\mathrm{V}$ sources, respectively. Firstly, GaAs substrates were immersed in poly-L-lysine (PLL) solution for $60 \mathrm{~s}$ and rinsed with deionized water before applying a droplet of colloidal solution of 30 or $50 \mathrm{~nm}$ diameter $\mathrm{Au}$ particles. After rinsing off the excessive Au colloidal particles with DI water, the wafers were blown dry using $\mathrm{N}_{2}$ gas and transferred into the MOCVD reactor chamber. For Si substrates, prior to this PLL treatment, a $\sim 50 \mathrm{~nm}$ thick GaAs buffer layer was deposited on Si using a two-temperature growth procedure: first grown at $400{ }^{\circ} \mathrm{C}$ with a V/III ratio of 15.4 for $60 \mathrm{~min}$, followed by a rapid $10 \mathrm{~min}$ growth at $700{ }^{\circ} \mathrm{C}$ with a V/III ratio of 154 . The buffer layer was then in situ annealed in an arsine atmosphere at $750{ }^{\circ} \mathrm{C}$ for $15 \mathrm{~min}$, then removed from the reactor for subsequent PLL treatment (details of this buffer growth process can be found in [28]). All $\mathrm{Au}$ decorated wafers were annealed in the MOCVD reactor under $\mathrm{AsH}_{3}$ at $600{ }^{\circ} \mathrm{C}$ for $10 \mathrm{~min}$, to desorb contaminants on the surface. Then, the reactor was cooled down to the desired temperature $\left(450{ }^{\circ} \mathrm{C}\right)$ to carry out the GaAs nanowire growth (referred to 'one-temperature procedure'). For the 'two-temperature procedure', growth initiated with a $1 \mathrm{~min}$ 'nucleation' step at the nucleation temperature, $T_{n}$, of $450^{\circ} \mathrm{C}$. The temperature was then rapidly ramped down to the subsequent growth temperature, $T_{g}$, of $375^{\circ} \mathrm{C}$ for further $30 \mathrm{~min}$ growth. For radial heterostructures, the reactor was heated up to $650{ }^{\circ} \mathrm{C}$ after the GaAs core growth for the subsequent $\mathrm{AlGaAs}$ or $\mathrm{GaP}$ shell layer growth. For axial heterostructures, InAs nanowire segment was grown at $450{ }^{\circ} \mathrm{C}$ and $\mathrm{GaSb}$ nanowire segment was grown at $425^{\circ} \mathrm{C}$.

The structural and crystallographic properties of nanowires were characterized by using field-emission scanning electron microscopy (FESEM) (Hitachi S4500) and transmission electron microscopy (TEM) (FEI Tecnai F30). For single nanowire micro-Raman, photoluminescence (PL) and time-resolved PL (TRPL) measurements, nanowires were transferred from the as-grown substrates to a Si substrate by rubbing the two substrate surfaces together. The PL spectra of individual nanowires were obtained at $15 \mathrm{~K}$ using slit confocal micro-PL spectroscopy. A $50 \times / 0.5$ NA long working distance microscope's objective was used to collect the PL from the nanowire sample and convey it to the spectrometer. For TRPL measurements, either an avalanche photodiode (APD) or a micro-channel photomultiplier tube (MCPT) is used to detect the PL signal. For Raman measurements, the nanowire was excited using a $100 \times / 0.7$ NA long working length objective with $532 \mathrm{~nm}$ laser excitation focused to a $0.7 \mu \mathrm{m}$ spot. The scattered light was dispersed using a $600 \mathrm{~mm}$ focal length DILOR xy triple spectrometer used in subtractive mode and detected by a liquid nitrogen-cooled CCD camera.

\section{Core-shell heterostructures}

Core-shell heterostructure nanowires attracted significant interest for optoelectronic, photonic or electronic devices $[10,13,29,30]$, since the shell can protect and passivate the active core nanowire surface, and provide carriers or engineer the bandgap structure if higher bandgap materials are used for the shell. However, the undesirable axial segment growth during the shell growth [31] and phase segregation [32, 33] still pose great challenges for obtaining perfect core-shell structures.

Core-shell heterostructures can be divided into two groups in terms of their lattice constant: lattice matched or mismatched. One typical example from each group will be presented as follows. 


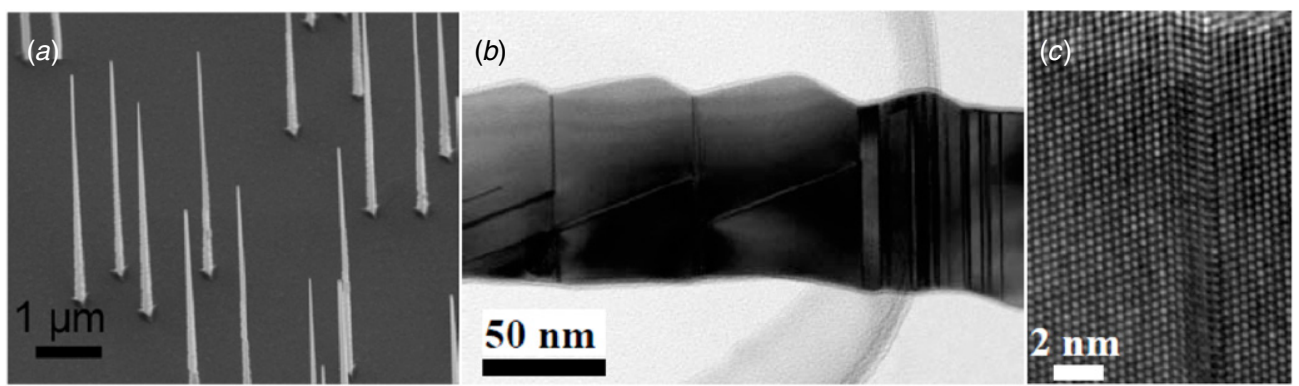

Figure 1. Morphology and structural characteristics of GaAs nanowires grown by one-temperature process: $(a)$ SEM image showing the highly tapered shape; $(b)$ TEM image showing high density of planar defects; $(c)$ a high resolution TEM displaying the twining defect.

\subsection{GaAs/AlGaAs core-shell nanowires}

Among various core-shell nanowires, GaAs/AlGaAs material system is probably the most popular one due to its considerable band offsets in conduction and valence bands, effective passivation, similar lattice constants, large difference of refractive index and high electron mobility [31, 32, 34-38]. In addition, GaAs/AlGaAs system has been widely used in industry for planar optoelectronic devices such as lasers and photodetectors. The mature processing techniques offer this material system a great advantage in device fabrication. In this paper, the great passivation effect from $\mathrm{AlGaAs}$ on GaAs nanowire surface is demonstrated.

We firstly grew GaAs nanowires at $450{ }^{\circ} \mathrm{C}$ using the one-temperature procedure. However, such nanowires show a zinc blende crystal phase with a high density of twins and stacking faults and significant tapering as shown in figure 1 [39]. These characteristics are common problems in various III-V semiconductor nanowires [40-42]. These defects can act as efficient nonradiative recombination centers in GaAs. In addition, due to the large surface recombination velocity $\left(5 \times 10^{6} \mathrm{~cm} \mathrm{~s}^{-1}\right)$ [43], the free GaAs surface is particularly efficient at quenching electron-hole pairs nonradiatively, a particularly acute problem in nanowires because of their large surface-area-to-volume ratio. Therefore, the PL emission from these defective GaAs nanowires is very weak even at low temperatures (figure $2(b)$ ). In order to increase their PL emission efficiency, an AlGaAs shell was grown to passivate the GaAs core (SEM image is shown in figure 2(a)). Our previous report from Titova et al has shown that this GaAs/AlGaAs core-shell structure increased the quantum efficiency of PL by two orders of magnitude over the bare GaAs nanowire [44]. However, the TRPL measurements still showed an extremely short carrier lifetime in single nanowires as shown in figure $3(d)$ (light-color spectrum), below the 80 ps system response time for detection by our timecorrelated photon counting system [45]. This short lifetime is attributed to two possible sources: one is the high density of twin defects and stacking faults in such a nanowire grown by one-temperature process as shown in figure $1(b)$, and the other is the possible oxidation of the AlGaAs shell. Oxygen is well known to be an extremely effective deep level in GaAs resulting in extremely short lifetimes [46]. In order to improve the carrier lifetime in this core-shell heterostructure, we
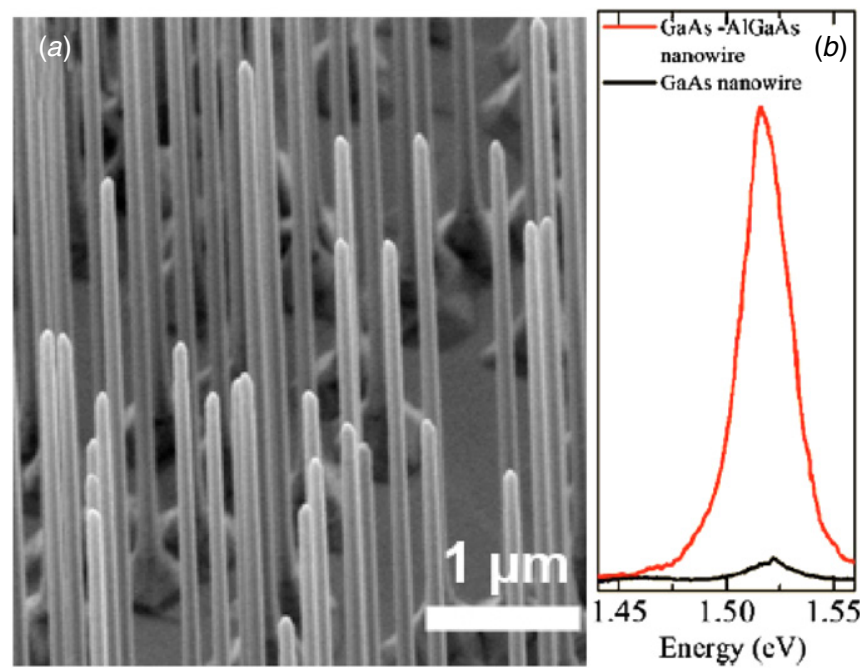

Figure 2. (a) SEM image of GaAs/AlGaAs core-shell nanowires grown by one-temperature process; $(b)$ PL spectra of the single bare GaAs and core-shell GaAs-AlGaAs nanowires, adapted from [22] with permission.

recently adopted a two-temperature growth procedure [47] to reduce (perhaps eliminate) the structural defects and tapering of GaAs nanowire core and, in addition, deposited a $5 \mathrm{~nm} \mathrm{GaAs}$ capping layer outside the $\mathrm{AlGaAs}$ shell to prevent the oxidation of AlGaAs. The SEM image of such a core-shell nanowire and TEM images of the defect-free GaAs nanowire are shown in figures 3(a)-(c). The two-temperature grown GaAs core with minimal tapered shape is displayed in figure $3(b)$ and the high resolution TEM (figure 1(c)) shows no planar defects in such a nanowire grown by two-temperature procedure.

The carrier lifetime in such core-shell nanowires improved dramatically, exhibiting a lifetime as long as $1 \mathrm{~ns}$ (the dark-color spectrum as shown in figure $3(d)$ ) which is comparable to very high quality 2D GaAs/AlGaAs wide DHSs and is consistent with an intrinsic exciton radiative recombination [48, 49]. Perera et al also showed that such a core-double shell nanowire heterostructure exhibits a higher quantum efficiency [50]. At high photoexcited carrier concentrations these nanowires also exhibit significant bandgap renormalization and state filling which allows us to determine the carrier density [50]. 

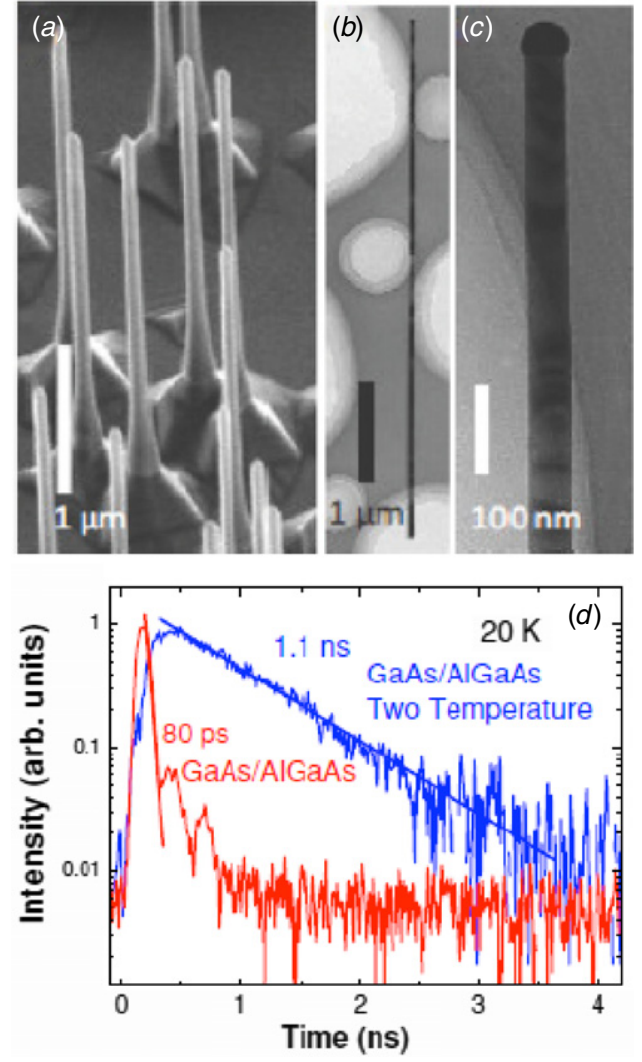

Figure 3. (a) SEM image of the GaAs/AlGaAs core-shell nanowires grown by two-temperature method; $(b)$ TEM image of a GaAs nanowire showing minimal tapering; $(c)$ a magnified TEM image displaying the absence of twin defects; $(d)$ time decays of PL from two single GaAs/AlGaAs core-shell nanowires. The short time decay ( $80 \mathrm{ps}$ ) is from the one-temperature grown GaAs nanowire core while the long exponential time decay (1.1 ns) is from the two-temperature growth GaAs nanowire core with an outer $5 \mathrm{~nm}$ GaAs cap to prevent oxidation of the AlGaAs shell. Adapted from $[23,25]$ with permission.

\subsection{GaAs/GaP core-shell nanowires}

Strain in core-shell nanowire structure is a critical factor to be considered in bandgap engineering for device applications. Unlike their bulk counterparts, the band offsets of core-shell nanowires can be significantly affected by the strain which has been demonstrated both experimentally and theoretically [51-54]. Recently, optimization of the strain in core-shell nanowires has been shown to enhance the device performance of $\mathrm{GaAs} / \mathrm{GaP}$ and $\mathrm{Si} / \mathrm{Ge}$ field emission transistors [55, 56]. However, the strain effects on band structure are difficult to be measured independently or decoupled from the quantum confinement effects [51, 54]. Here, we show a consistent picture between strain and band structure by direct measurements using Raman scattering and PL, respectively, on the same single GaAs/GaP nanowire.

The SEM and TEM images of the GaAs/GaP core-shell nanowires are shown in figures $4(a)-(c)$. Si surface was fully covered by the GaAs buffer layer which forms continuous islands due to the strain between GaAs and $\mathrm{Si}$ (as shown in figure 2(a)). GaAs/GaP core-shell nanowires nucleated on top of GaAs buffer layer and grew perpendicularly to the $\mathrm{Si}$ (1 11 1) substrate. The TEM image (figure $4(b)$ ) of the bottom region of a nanowire was taken along the [ 1112$]$ zone axis as indicated by the arrow in figure 4(c) to maximize the strain contrast between $\mathrm{GaAs}$ and $\mathrm{GaP}$ due to the lattice mismatch of the materials $(\sim 3.6 \%)$. The GaAs core is identified by the moiré fringes in this side view TEM image and can be more accurately identified using cross-sectional TEM (XTEM) as shown in figure $4(c)$. The GaAs core is estimated to be $\sim 50 \mathrm{~nm}$ and the GaP shell is estimated to be $\sim 25 \mathrm{~nm}$. The strain contrasts can also be identified at the $\mathrm{GaAs} / \mathrm{GaP}$ interface in figure 4(c). Selected area electron diffraction (SAED) (not shown here) confirmed that both the GaAs nanowire core and GaP nanowire shell have the cubic zinc blende structure.

The stress in this material system can be measured by Raman scattering from phonons. We have carried out Raman scattering from three individual $\mathrm{GaAs} / \mathrm{GaP}$ nanowires at room temperature in the backscattering geometry (as shown in figure 5) [52]. The Raman spectrum of an unstrained GaAs epilayer grown on a GaAs substrate is also shown as a reference. Compared to the transverse optical (TO) and longitudinal optical (LO) phonon energies of the epitaxial $\mathrm{GaAs}$, the GaAs/GaP core-shell nanowires displayed a significant shift of GaAs TO phonon and LO phonon energies to higher frequencies $\left(\sim 10 \mathrm{~cm}^{-1}\right)$, indicating significant compressive stress in the GaAs core. In contrast, the GaP TO phonon and LO phonon energies show a significant downward

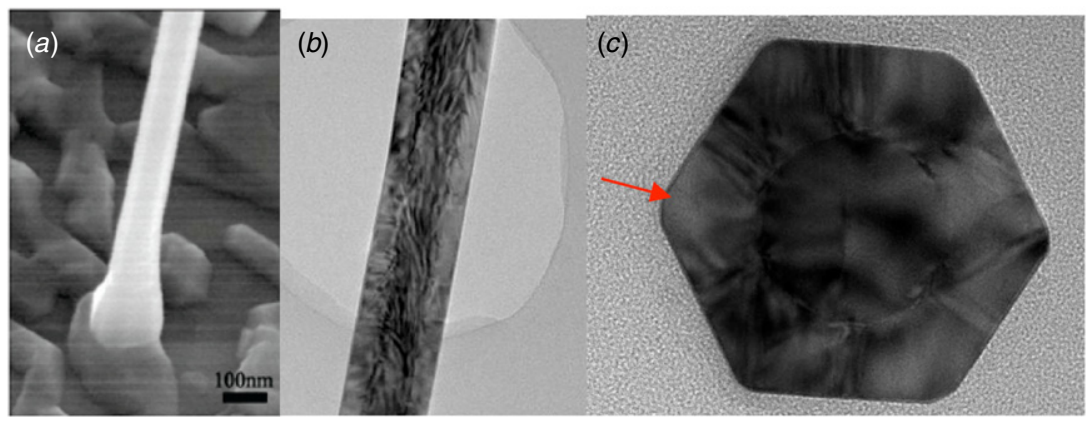

Figure 4. (a) SEM image of a GaAs/GaP nanowire on Si substrate with GaAs buffer. (b) TEM image taken from [1 12 2] zone axis showing the side view of the bottom section of a nanowire. $(c)$ A typical cross-sectional TEM (XTEM) image viewing along the [1 111$]$ zone axis of the bottom region of a nanowire, showing a strained core-shell structure. The arrow indicates the viewing direction of [ $\left[\begin{array}{lll}1 & 1 & 2\end{array}\right]$ for the side view image $(b)$. Adapted from [27] with permission. 


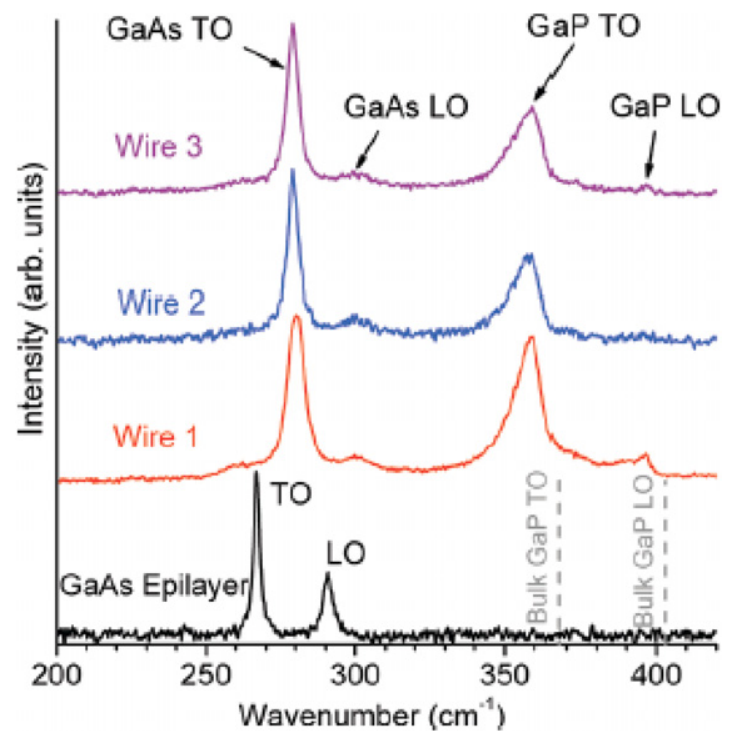

Figure 5. Raman spectra of GaAs/GaP nanowires (light-color lines) and GaAs epilayer (dark-color line) at room temperature. The dashed lines show TO and LO energies of GaP. Adapted from [29] with permission.

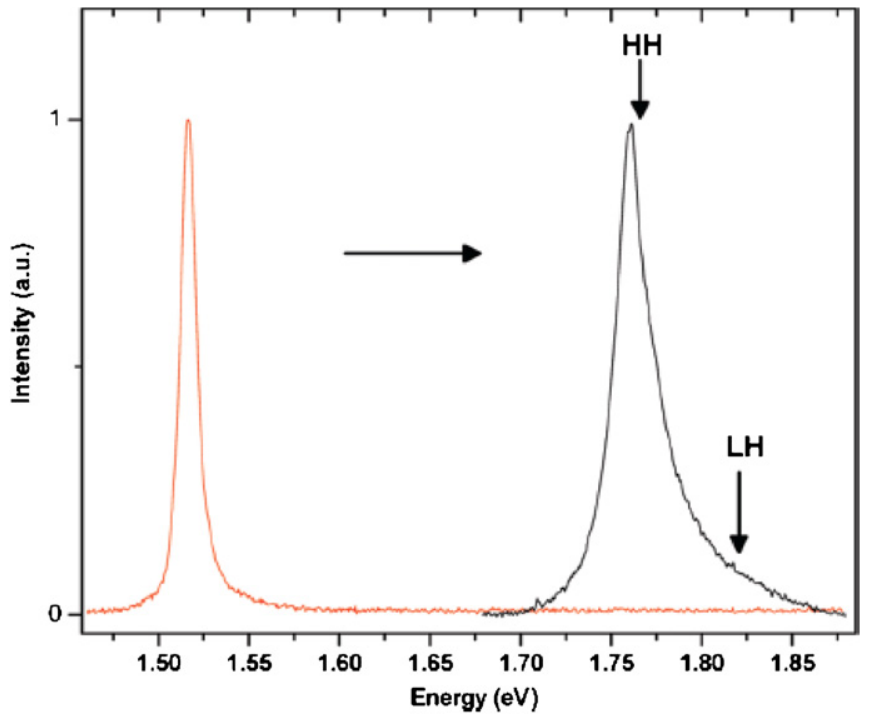

Figure 6. Low-temperature PL spectrum of GaAs/AlGaAs (left spectrum) and $\mathrm{GaAs} / \mathrm{GaP}$ (right spectrum) core-shell nanowires. The vertical arrows show energies of $\mathrm{HH}$ and LH bandgap, respectively, predicted from Raman measurement. Adapted from [29] with permission.

Raman shift, indicating a tensile stress in the GaP shell. In addition, the low-energy tail of GaP TO phonon response may indicate a strain gradient in the shell. The hydrostatic and shear strain of the GaAs core have been calculated from the Raman spectra and showed good agreement with the theoretical predictions [52, 53, 57].

These large hydrostatic and shear strains in the GaAs core are expected to result in large effects in electronic states. To confirm this, micro-PL measurements were performed on single $\mathrm{GaAs} / \mathrm{GaP}$ nanowires. A typical spectrum is shown in figure 6 together with a reference PL spectrum from a high quality $\mathrm{GaAs} / \mathrm{AlGaAs}$ nanowire measured earlier

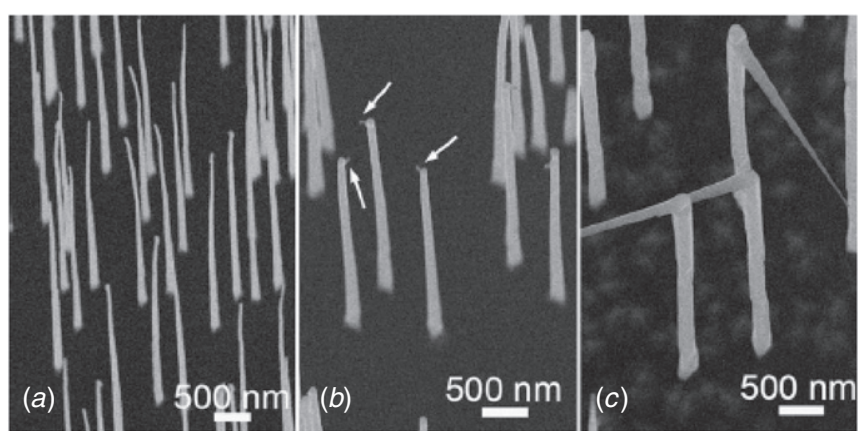

Figure 7. SEM images of GaAs/InAs nanowires with different InAs growth times. $(a)-(c)$ correspond to 1, 5 and $30 \mathrm{~min}$ InAs growth, respectively. The images were taken when the substrate normal was tilted $20^{\circ}$ away from the electron beam. Adapted from [32] with permission.

(figure 1(a)). The PL emission energy from GaAs core in GaAs/GaP nanowires clearly shifts to higher energies by $\sim 260 \mathrm{meV}$ from the expected $1.515 \mathrm{eV}$ GaAs-free exciton peak observed in GaAs/AlGaAs nanowires. The vertical arrows show the expected emission energies for the heavy hole $(\mathrm{HH})$ and light hole $(\mathrm{LH})$ excitons based on the strain information calculated from the Raman spectra. The excellent agreement between the experimental data and theoretical calculation indicates that the strain obtained from the Raman measurement can be used to predict bandgap changes and the HH-LH splitting.

\section{Axial heterostructures}

In addition to core-shell geometry, nanowire heterostructures can also form axially along its length-so-called axial heterostructure nanowires. As mentioned earlier in the introduction, nanowires offer great advantage over their bulk counterparts in forming defect-free and sharp heterojunctions from highly lattice-mismatched materials [19-23]. In order to form DHS confinement in nanowires, the axial heterostructures must grow in a straight form and have high quality interfaces in both interface directions. However, this is not always true for axial heterostructure nanowires. In the following sections, we present two examples: InAs on $\mathrm{GaAs}$ and $\mathrm{GaSb}$ on GaAs, where one cannot form straight heterostructure nanowires ( $\mathrm{GaAs} / \mathrm{InAs}$ ) but the other one can (GaAs/GaSb).

\subsection{GaAs/InAs axial nanowires}

Although GaAs/InAs two-dimensional (2D) heterostructures are one of the key material systems for optoelectronic device applications, their 1D counterparts are much less studied $[24,25,58-64]$ in spite of their potential optoelectronic and electronic applications. Not being able to grow InAs nanowires straight on GaAs nanowires is probably the most difficult challenge to be overcome to form DHS [24, 25, 59]. Paladugu et al have used TEM to investigate the growth mechanism of GaAs/InAs axial nanowire heterostructures in detail [25, 59-63]. Figure 7 shows SEM images taken 


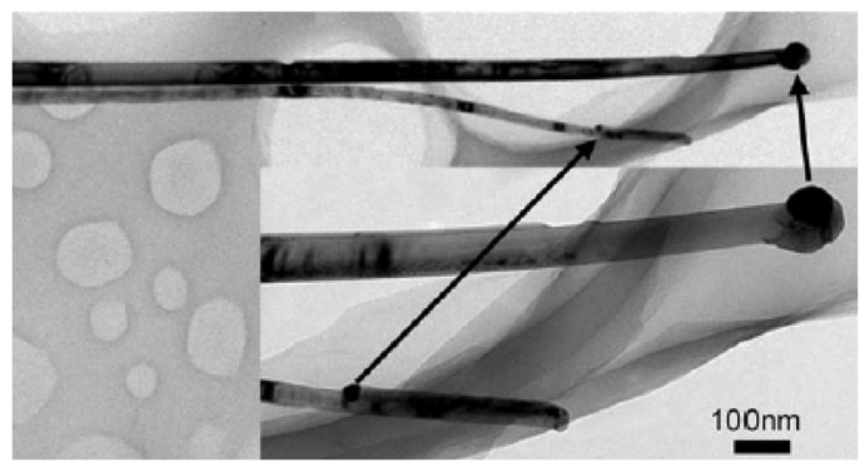

Figure 8. A TEM image showing the mixed-sized nanowires: the thicker nanowire tends to be straight, while the thinner one tends to be bent. Adapted from [15] with permission.

from GaAs/InAs axial nanowire heterostructures grown with different growth times (1 min, 5 min and $30 \mathrm{~min}$ ) for the InAs sections. A small branch-like structure can be found near the tip of GaAs/InAs nanowires with 5 min growth of InAs nanowire sections, as indicated by arrows in figure $7(b)$, but no clear branch is presented in nanowires with 1 min growth of the InAs (see figure 7(a)). In contrast, in the case of $30 \mathrm{~min}$ growth of InAs (figure 7(c)), each nanowire displays a welldeveloped branch and the overall morphology of the nanowires resembles a stem-branch nanostructure.

To determine how and why this branching phenomenon occurs, a detailed TEM investigation was firstly carried out on nanowires where InAs growth had been initiated for just $1 \mathrm{~min}$. Although the nanowire growth was initiated by $\sim 30 \mathrm{~nm}$ Au particles, the TEM analysis showed a significant number of thicker nanowires, with diameters up to $90 \mathrm{~nm}$, probably due to the coalescence of Au particles during the annealing stage at $600{ }^{\circ} \mathrm{C}$. A typical example of these mixed-size nanowires is shown in figure 8 where different morphologies can be seen: for thinner nanowires, the Au particle has moved away from the nanowire tip down to the sidewall while only sideward movement of the Au particle was observed for the thicker ones [25]. Fortunately, these thicker nanowires provide us with an opportunity to investigate the initial stage of the InAs nanowire growth. Figures $9(a)-(c)$ are TEM images of such nanowires showing that the $\mathrm{Au}$ particles are just beginning to move sideward from the tip position (GaAs/InAs interfaces are marked by arrows) in three different stages.

To understand the growth difference between InAs on GaAs nanowires (leading to a clustered InAs) and GaAs on InAs nanowires (resulting in straight nanowires as evidenced in $[24,58,63]$ ), we adopt the model proposed for description of heterogeneous nucleation of a solid from a metallic liquid along a solid surface of a mold [65]. This model is used because it is reasonable to assume in this study that the $\mathrm{Au}$ particle is in the liquid form during nanowire growth and GaAs and InAs can act as the wall of the mold and nucleated solid, respectively. Figure $9(d)$ shows a schematic diagram of clustered InAs formed between the $\mathrm{Au}$ droplet and GaAs, which is similar to the morphology shown in figure $9(a)$. At equilibrium, the model gives $\gamma_{A u-G a A s}=$ $\gamma_{I n A s-G a A s}+\gamma_{A u-I n A s} \cdot \operatorname{COS} \delta$, where $\gamma_{A-B}$ is the interfacial
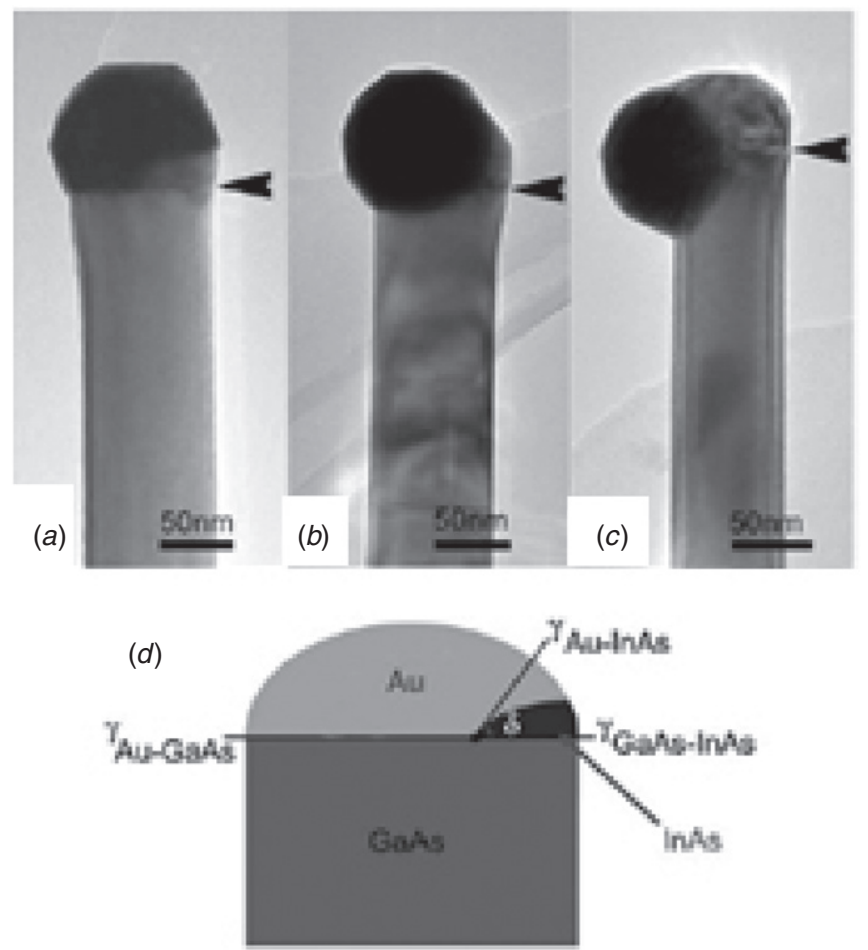

Figure 9. (a)-(c) TEM images showing initial stages of InAs growth on GaAs nanowires. $(d)$ A HRTEM image showing the atomic structure of the initially grown InAs in $(b)$. (e) A schematic diagram showing the interfaces between clustered InAs, GaAs and $\mathrm{Au}$ at their contact point. Adapted from [15] with permission.

energy between the material A and the material B and $\gamma$ is the contact angle between the clustered InAs and the GaAs surface. The interfacial energy of Au-GaAs is assumed to be less than Au-InAs; therefore, $\gamma>0$ (clustering occurred) when InAs grows on GaAs resulting in InAs clustering at the edge and later sideward movement. While in the case of GaAs grown on InAs nanowires, straight nanowire heterostructures were obtained through layer-by-layer growth of GaAs, i.e. $\gamma=$ 0 . Similar morphology in heterostructure nanowires was also reported by Dick et al and was explained in terms of interfacial energies as well [66].

Once the Au particle has moved to the sidewalls of the GaAs nanowire (figures 8 and $9(c)$ ), continuing sideways growth of InAs may be expected. However, as shown in figures $7(b)$ and $(c)$, InAs nanowires formed branches on the GaAs nanowire stem instead. To determine the mechanism for this branched morphology, further TEM investigations were conducted on nanowires with longer growth time of InAs sections. Figures $10(a)-(d)$ show TEM images taken from the top portion of nanowire with $1 \mathrm{~min}, 3 \mathrm{~min}, 5 \mathrm{~min}$ and $30 \mathrm{~min}$ growth of InAs section. Note that the nanowire in figure $10(a)$ is the thinner one shown in figure 8. As shown in figure 10(a), after 1 min growth of InAs section, the Au particle has moved away from the GaAs nanowire tip and migrated down the GaAs nanowire sidewall. However, this downward sliding of $\mathrm{Au}$ particle has been retarded by radial growth of InAs around the GaAs nanowire which can be identified by the moire fringes and the strain contrast along the nanowire in figure $10(b)$ with 3 min growth of InAs. Thus the Au particle 


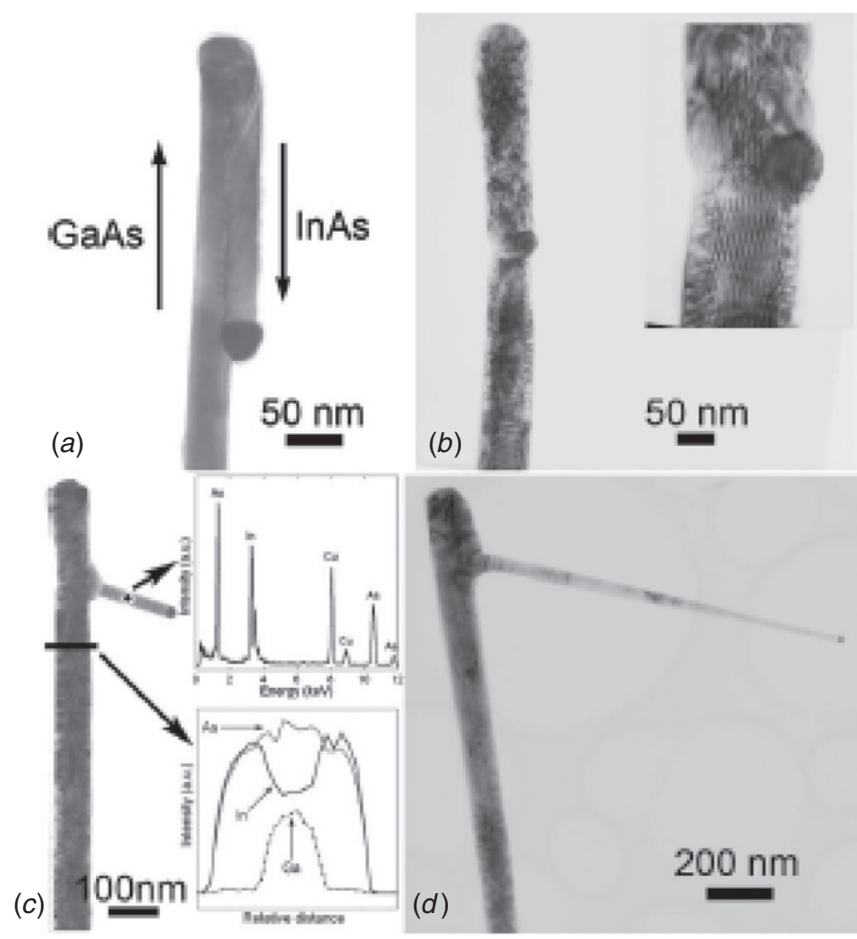

Figure 10. TEM images of GaAs/InAs nanowires with different InAs growth times. $(a)-(d)$ correspond to $1,3,5$ and 30 min InAs growth, respectively. The inset in $(b)$ is the magnified image of the nanowire portion at the Au nanoparticle. The insets in $(c)$ show the results of the EDS analyses at different locations of the nanowire and the $\mathrm{Cu}$ peak is from the TEM grid. Adapted from [32] with permission.

remains near the GaAs nanowire top region. Generally, in $\mathrm{Au}$-assisted growth of nanowires, radial growth is related to a higher temperature process [67]. However, significant InAs radial growth has taken place even at $450{ }^{\circ} \mathrm{C}$ in this work similar to the case of GaP/GaAs [67]. With further supply of In and As vapor sources, the InAs nanowire started to 'shoot out' along [1 111 ] direction as a branch on GaAs, or more accurately, a GaAs/InAs core-shell nanowire stem as shown in figures $10(c)$ and $(d)$. Energy dispersive spectroscopy (EDS) analysis further confirms that the branch is composed of InAs and that the stem has a core-shell structure of GaAs and InAs, as shown in the insets of figure $10(c)$. With further growth, this InAs nanowire branch will continue to grow as a standalone nanowire while the InAs shell grows thicker at the same time, resulting in a thicker GaAs/InAs core-shell nanowire stem and a well-developed InAs nanowire branch, as shown in figure $10(d)$.

\subsection{GaAs/GaSb axial nanowires}

GaSb-based III-V semiconductors are of great interest for near- and mid-infrared optoelectronic and high-speed electronic devices. Though it is very challenging to grow high quality $\mathrm{GaSb}$ epilayers on GaAs due to the large lattice mismatch $(\sim 7.8 \%)$, nanowires offer the possibility of integrating GaSb with more 'classic' GaAs. Understanding of the growth mechanism and growth control of $\mathrm{GaAs} / \mathrm{Ga}(\mathrm{As}) \mathrm{Sb}$ heterostructure nanowires have advanced significantly since

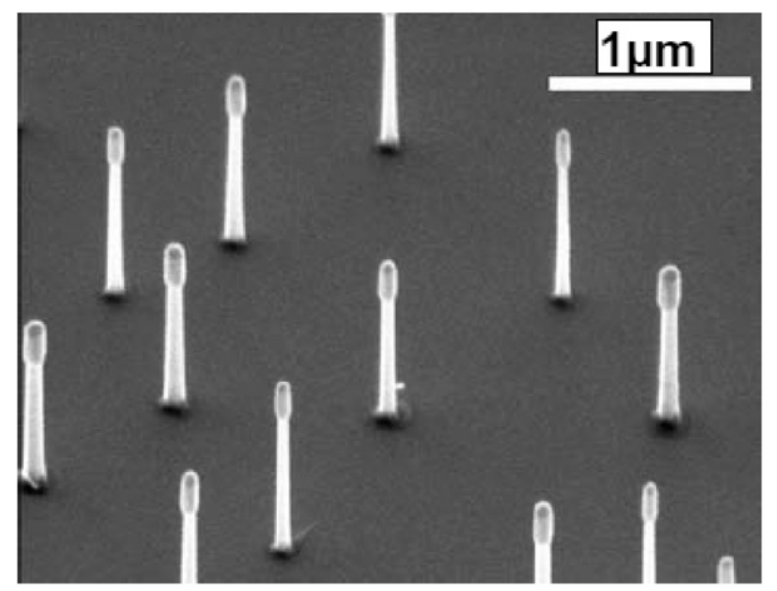

Figure 11. SEM image of GaAs/GaSb nanowire heterostructures. The substrate normal was tilted $45^{\circ}$ away from the electron beam.

GaSb nanowire lasers were first demonstrated by Chin et al [68] and defect-free GaSb nanowires on GaAs nanowires were first reported by Guo et al in 2006 [21]. More recently, GaAs/GaAsSb/GaAs DHS nanowires have been demonstrated [69, 70], indicating one more step closer to realizing operational devices based on this material system.

The general morphology of such a heterostructure grown by our group is shown in figure 11. In contrast to GaAs/InAs nanowire heterostructures, all $\mathrm{GaAs} / \mathrm{GaSb}$ nanowires are straight and well aligned in a vertical direction on GaAs ( $\left.\begin{array}{lll}1 & 1 & 1\end{array}\right)$ substrate. Each individual nanowire has a tapered GaAs body with a thick column-shaped GaSb head. The length of the entire nanowire is $\sim 1 \mu \mathrm{m}$ and the diameter of heads is in the range of several tens of nanometers.

TEM was carried out to determine detailed microstructure of these GaAs/GaSb nanowire heterostructures. Figure 12(a) is a bright-field TEM image and shows a typical top section of the nanowire containing GaAs (thin body), GaSb (thick head), as well as the Au catalyst (top hemispherical particle). It is interesting to note that the diameter of the GaSb segment is about $40 \%$ thicker than the GaAs part. This significant change in diameter was first explained as the strain relaxation via lateral expansion [21]. Though this lateral expansion still exists, it is more reasonable to believe that the composition change in Au eutectic alloy (i.e. more Ga incorporated in Au or $\mathrm{Sb}$ addition to form $\mathrm{Au}(\mathrm{GaSb})_{2}$ or $\mathrm{Au}(\mathrm{GaSb})$ types of phases) are accountable for the majority of this diameter increase [71, 72]. SAED taken from the interface between GaSb and GaAs (figure $12(b)$ ) clearly shows two sets of $\left\langle\begin{array}{lll}1 & 1 & 0\end{array}\right\rangle$ patterns with one set being GaAs and the other being GaSb. A $7.5 \pm$ $0.3 \%$ lattice mismatch was determined by the measurement of corresponding atomic planes for GaAs and $\mathrm{GaSb}$ indicating a fully relaxed $\mathrm{GaSb}$ nanowire, since the lattice mismatch between unstrained GaAs and GaSb is $7.8 \%$.

Although many planar defects can be clearly seen in the GaAs region (indicated by arrows in figure 12(c)), the $\mathrm{GaSb}$ region remains defect-free (figure $12(d)$ ). To determine whether misfit dislocations exist at the $\mathrm{GaAs} / \mathrm{GaSb}$ interface, a high resolution TEM investigation was carried out as shown in figure 12(e). Although the GaAs/GaSb interface is not 


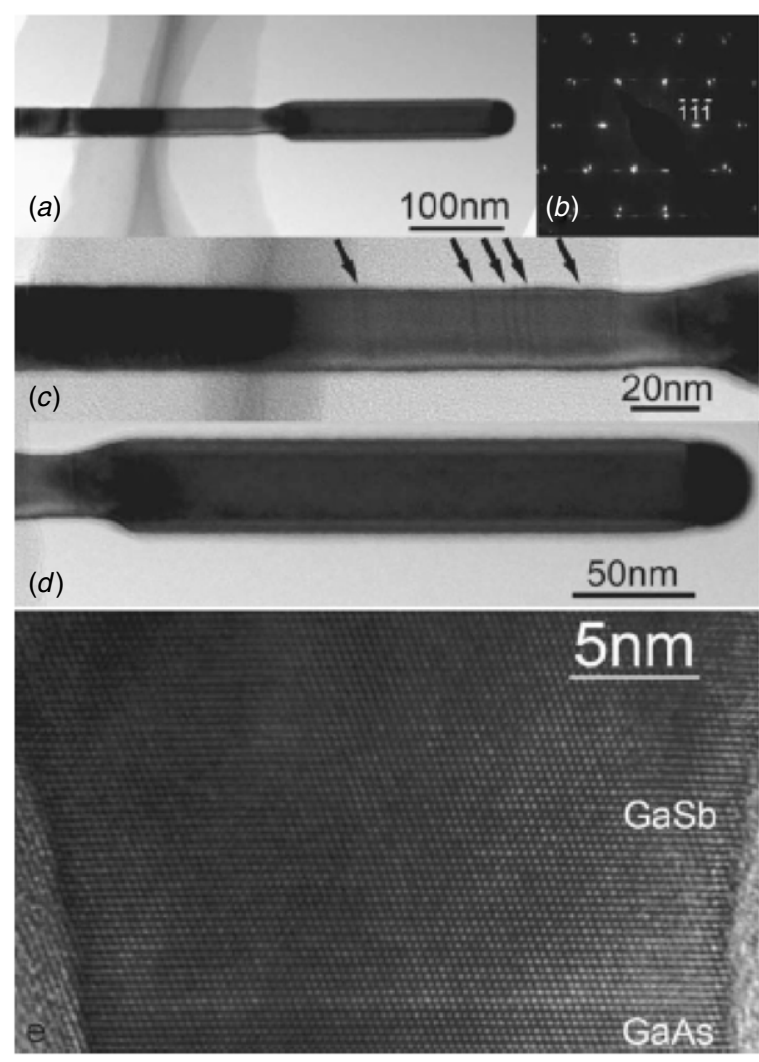

Figure 12. (a) A TEM image taken from the top section of a $\mathrm{GaSb} / \mathrm{GaAs}$ nanowire heterostructure. (b) A SAED pattern showing two sets of well-distinguished $\left\langle\begin{array}{lll}1 & 1 & 0\end{array}\right)$ electron diffraction patterns. (c) A TEM image of the GaAs section showing many planar defects (marked by arrows). (d) A TEM image of the GaSb section showing no lattice defects. (e) A high-resolution image (the growth direction pointing upside) in the $\mathrm{GaSb} / \mathrm{GaAs}$ interfacial region showing no misfit dislocations in the region. Adapted from [13] with permission.

clearly identified from figure 12(e), no misfit dislocations exist in this interfacial region. This suggests that the entire strain relaxation for the GaSb nanowire grown is through the lateral expansion [21] without introducing any misfit dislocations.

\section{Summary and conclusions}

In summary, we have reviewed the morphology, microstructure and optical properties of various GaAs nanowire-based heterostructures including $\mathrm{GaAs} / \mathrm{AlGaAs}$ and $\mathrm{GaAs} / \mathrm{GaP}$ core-shell structures, and GaAs/InAs and GaAs/GaSb axial structures. We found that the suppression of surface/impurityrelated non-radiative combination pathways is critical for the carrier lifetime in GaAs nanowires. The strain between the core and shell can be utilized to engineer the electronic structure of the GaAs nanowire core. For axial heterostructure nanowires, the metal-semiconductor surface energy is believed to be one of the determining factors for the growth direction - whether straight or branched structures. Defect-free GaSb nanowires were achieved axially on GaAs nanowires confirming that heterostructure nanowires are strong candidates to overcome the lattice mismatch problems in conventional 2D semiconductor structures.

\section{Acknowledgments}

We acknowledge the financial support of the Australian Research Council and the US National Science Foundation through grants 0806700, 0806572 and 0701703. Facilities used in this work are supported by the Australian National Fabrication Facility. We acknowledge the essential efforts of our colleagues and the graduate students who have made this research possible, including Hannah Joyce, Suriati Paiman, Jung Hyun Kang, Yong Kim, Mohan Paladugu, Yanan Guo, Xin Zhang, Thang Ba Hoang, Lyuba Titova, Saranga Perera, Melodie Fickenscher, Karunananda Pemasiri, Parveen Kumar and Mohammad Montazeri.

\section{References}

[1] Alferov Z I and Kazarinov R F 1963 Semiconductor laser with electric pumping USSR Patent No. 181737

[2] Kroemer H 1963 A proposed class of heterojunction injection lasers Proc. IEEE $\mathbf{5 1} 1782$

[3] Czaban J A, Thompson D A and LaPierre R R 2009 GaAs core-shell nanowires for photovoltaic applications Nano Lett. 9 148-54

[4] Heinrich J et al 2010 Single photon emission from positioned GaAs/AlGaAs photonic nanowires Appl. Phys. Lett. 9621117

[5] Minot E D et al 2007 Single quantum dot nanowire LEDs Nano Lett. 7 367-71

[6] Borgstrom M T, Zwiller V, Muller E and Imamoglu A 2005 Optically bright quantum dots in single nanowires Nano Lett. 5 1439-43

[7] Pettersson H, Tragardh J, Persson A I, Landin L, Hessman D and Samuelson L 2006 Infrared photodetectors in heterostructure nanowires Nano Lett. 6 229-32

[8] Wang J F, Gudiksen M S, Duan X F, Cui Y and Lieber C M 2001 Highly polarized photoluminescence and photodetection from single indium phosphide nanowires Science 293 1455-7

[9] Patolsky F, Zheng G and Lieber C M 2006 Nanowire sensors for medicine and the life sciences Nanomedicine 1 51-65

[10] Qian F, Gradecak S, Li Y, Wen C Y and Lieber C M 2005 Core/multishell nanowire heterostructures as multicolor, high-efficiency light-emitting diodes Nano Lett. 5 2287-91

[11] Hua B, Motohisa J, Kobayashi Y, Hara S and Fukui T 2009 Single GaAs/GaAsP coaxial core-shell nanowire lasers Nano Lett. 9 112-6

[12] Johnson J C, Choi H J, Knutsen K R, Schaller R D, Yang P D and Saykally R J 2002 Single gallium nitride nanowire lasers Nat. Mater. 1 106-10

[13] Gradecak S, Qian F, Li Y, Park H G and Lieber C M 2005 GaN nanowire lasers with low lasing thresholds Appl. Phys. Lett. 8773111

[14] Fuhrer A, Fasth C and Samuelson L 2007 Single electron pumping in InAs nanowire double quantum dots Appl. Phys. Lett. 9152109

[15] Wagner R S and Ellis W C 1964 Vapor-liquid-solid mechanism of single crystal growth Appl. Phys. Lett. 4 89-90

[16] Persson A I, Naylor A S, Jonsdottir I H, Nyberg F, Eriksson P S and Thorlin T 2004 Differential regulation of hippocampal progenitor proliferation by opioid receptor antagonists in running and non-running spontaneously hypertensive rats Eur. J. Neurosci. 19 1847-55

[17] Dick K A, Deppert K, Martensson T, Mandl B, Samuelson L and Seifert W 2005 Failure of the vapor-liquid-solid mechanism in Au-assisted MOVPE growth of InAs nanowires Nano Lett. 5 761-4 
[18] Zervos M and Feiner L F 2004 Electronic structure of piezoelectric double-barrier InAs/InP/InAs/ InP/InAs( 1111$)$ nanowires $J$. Appl. Phys. 95 281-91

[19] Borgstrom M T, Verheijen M A, Immink G, de Smet T and Bakkers E 2006 Interface study on heterostructured GaP-GaAs nanowires Nanotechnology 17 4010-3

[20] Bjork M T et al 2002 One-dimensional heterostructures in semiconductor nanowhiskers Appl. Phys. Lett. 80 1058-60

[21] Guo Y N et al 2006 Structural characteristics of GaSb/GaAs nanowire heterostructures grown by metal-organic chemical vapor deposition Appl. Phys. Lett. 89231917

[22] Gudiksen M S, Lauhon L J, Wang J, Smith D C and Lieber C M 2002 Growth of nanowire superlattice structures for nanoscale photonics and electronics Nature 415 617-20

[23] Caroff P et al 2008 High-quality InAs/InSb nanowire heterostructures grown by metal-organic vapor-phase epitaxy Small 4 878-82

[24] Dick K A et al 2007 The morphology of axial and branched nanowire heterostructures Nano Lett. 7 1817-22

[25] Paladugu M et al 2007 Novel growth phenomena observed in axial InAs/GaAs nanowire heterostructures Small 3 1873-7

[26] Lauhon L J, Gudiksen M S and Lieber C M 2004 Semiconductor nanowire heterostructures Phil. Trans. $R$. Soc. A 362 1247-60

[27] Mieszawska A J, Jalilian R, Sumanasekera G U and Zamborini F P 2007 The synthesis and fabrication of one-dimensional nanoscale heterojunctions Small 3 722-56

[28] Kang J H et al 2010 Novel growth and properties of GaAs nanowires on Si substrates Nanotechnology 2135604

[29] Li Y, Qian F, Xiang J and Lieber C M 2006 Nanowire electronic and optoelectronic devices Mater. Today 9 18-27

[30] Bryllert T, Wernersson L E, Froberg L E and Samuelson L 2006 Vertical high-mobility wrap-gated InAs nanowire transistor IEEE Electron Device Lett. 27 323-5

[31] Chen C, Braidy N, Couteau C, Fradin C, Weihs G and LaPierre R 2008 Multiple quantum well AlGaAs nanowires Nano Lett. 8 495-9

[32] Skold N et al 2006 Phase segregation in AllnP shells on GaAs nanowires Nano Lett. 6 2743-7

[33] Chen C, Shehata S, Fradin C, LaPierre R, Couteau C and Weihs G 2007 Self-directed growth of AlGaAs core-shell nanowires for visible light applications Nano Lett. 7 2584-9

[34] Noborisaka J, Motohisa J, Hara S and Fukui T 2005 Fabrication and characterization of freestanding $\mathrm{GaAs} / \mathrm{AlGaAs}$ core-shell nanowires and $\mathrm{AlGaAs}$ nanotubes by using selective-area metalorganic vapor phase epitaxy Appl. Phys. Lett. 8793109

[35] Ouattara L et al 2007 GaAs/AlGaAs nanowire heterostructures studied by scanning tunneling microscopy Nano Lett. 7 2859-64

[36] Tambe M J, Lim S K, Smith M J, Allard L F and Gradecak S 2008 Realization of defect-free epitaxial core-shell GaAs/AlGaAs nanowire heterostructures Appl. Phys. Lett. 93 51917-

[37] Tateno K, Gotoh H and Watanabe Y 2006 Multi-quantum structures of GaAs/AlGaAs free-standing nanowires Japan. J. Appl. Phys. 45 3568-72

[38] Wu Z H, Sun M, Mei X Y and Ruda H E 2004 Growth and photoluminescence characteristics of AlGaAs nanowires Appl. Phys. Lett. 85 657-9

[39] Zou J et al 2007 Growth mechanism of truncated triangular III-V nanowires Small 3 389-93

[40] Hiruma K et al 1993 GaAs free-standing quantum-size wires J. Appl. Phys. 74 3162-71

[41] Borgstrom M, Deppert K, Samuelson L and Seifert W 2004 Size- and shape-controlled GaAs nano-whiskers grown by MOVPE: a growth study J. Cryst. Growth. 260 18-22
[42] Johansson J et al 2006 Structural properties of (1 11 1)B-oriented III-V nanowires Nat. Mater. 5 574-80

[43] Lloyd-Hughes J et al 2006 Influence of surface passivation on ultrafast carrier dynamics and terahertz radiation generation in GaAs Appl. Phys. Lett. 89232102

[44] Titova L V et al 2006 Temperature dependence of photoluminescence from single core-shell GaAs-AlGaAs nanowires Appl. Phys. Lett. 89173126

[45] Smith L M, Jackson H E, Yarrison-Rice J M and Jagadish C 2010 Insights into single semiconductor nanowire heterostructures using time-resolved photoluminescence Semicond. Sci. Technol. 25024010

[46] Ryan J M, Huang J W, Kuech T F and Bray K L 1994 The effects of temperature and oxygen concentration on the photoluminescence of epitaxial metalorganic vapor-phase epitaxy GaAs:O J. Appl. Phys. 76 1175-9

[47] Joyce H J et al 2007 Twin-free uniform epitaxial GaAs nanowires grown by a two-temperature process Nano Lett. 7 921-6

[48] Nelson R J and Sobers R G 1978 Minority-carrier lifetimes and internal quantum efficiency of surface-free GaAs J. Appl. Phys. 49 6103-8

[49] Wolford D J et al 1991 Intrinsic recombination and interface characterization in 'surface-free' GaAs structures $J$. Vac. Sci. Technol. B 9 2369-76

[50] Perera S et al 2008 Nearly intrinsic exciton lifetimes in single twin-free GaAs/AlGaAs core-shell nanowire heterostructures Appl. Phys. Lett. 9353110

[51] Skold N et al 2005 Growth and optical properties of strained GaAs-GaxIn1-xP core-shell nanowires Nano Lett. 5 1943-7

[52] Montazeri M et al 2010 Direct measure of strain and electronic structure in GaAs/GaP core-shell nanowires Nano Lett. 10 880-6

[53] Pistol M E and Pryor C E 2008 Band structure of core-shell semiconductor nanowires Phys. Rev. B 78115319

[54] Zanolli Z, Pistol M E, Froberg L E and Samuelson L 2007 Quantum-confinement effects in InAs-InP core-shell nanowires J. Phys.: Condens. Matter 1995219

[55] He Y H, Zhao Y N, Fan C K, J F, Han R Q and Liu X Y 2009 IEEE Trans. Electron Devices 561199

[56] Trammell T E, Zhang X, Li Y L C, L Q and Dickey E C 2008 J. Cryst. Growth 310 3084-92

[57] Pistol M E and Pryor C E 2009 Band structure of segmented semiconductor nanowires Phys. Rev. B 80035316

[58] Hiruma K, Murakoshi H, Yazawa M and Katsuyama T 1996 Self-organized growth of GaAs/InAs heterostructure nanocylinders by organometallic vapor phase epitaxy J. Cryst. Growth. 163 226-31

[59] Paladugu $\mathrm{M}$ et al 2007 Evolution of InAs branches in InAs/GaAs nanowire heterostructures Appl. Phys. Lett. 9133115

[60] Paladugu $\mathrm{M}$ et al 2008 Polarity driven formation of InAs/GaAs hierarchical nanowire heterostructures Appl. Phys. Lett. 931908

[61] Paladugu M et al 2009 Crystallographically driven Au catalyst movement during growth of InAs/GaAs axial nanowire heterostructures J. Appl. Phys. 10573503

[62] Paladugu M et al 2009 Formation of hierarchical InAs Nanoring/GaAs nanowire heterostructures Angew. Chem., Int. Ed. 48 780-3

[63] Paladugu $\mathrm{M}$ et al 2008 Nature of heterointerfaces in $\mathrm{GaAs} / \mathrm{InAs}$ and InAs/GaAs axial nanowire heterostructures Appl. Phys. Lett. 931911

[64] Ohlsson B J et al 2002 Growth and characterization of GaAs and InAs nano-whiskers and InAs/GaAs heterostructures Physica E 13 1126-30

[65] Chan R W 1970 Physical Metallurgy 2nd edn (Amsterdam: Elsevier) pp 406-8 
[66] Dick K A et al 2007 The morphology of axial and branched nanowire heterostructures Nano Lett. 7 1817-22

[67] Verheijen M A, Immink G, de Smet T, Borgstrom M T and Bakkers E 2006 Growth kinetics of heterostructured GaP-GaAs nanowires J. Am. Chem. Soc. 128 1353-9

[68] Chin A H, Vaddiraju S, Maslov A V, Ning C Z, Sunkara M K and Meyyappan M 2006 Near-infrared semiconductor subwavelength-wire lasers Appl. Phys. Lett. 8863115

[69] Dheeraj D L et al 2008 Zinc blende GaAsSb nanowires grown by molecular beam epitaxy Nanotechnology 1975605
[70] Plissard S, Dick K A, Wallart X and Caroff P 2010 Gold-free $\mathrm{GaAs} / \mathrm{GaAsSb}$ heterostructure nanowires grown on silicon Appl. Phys. Lett. 96121901

[71] Weng X J, Burke R A, Dickey E C and Redwing J M 2010 Effect of reactor pressure on catalyst composition and growth of GaSb nanowires J. Cryst. Growth. 312 514-9

[72] Jeppsson M et al $2008 \mathrm{GaAs} / \mathrm{GaSb}$ nanowire heterostructures grown by MOVPE J. Cryst. Growth. 310 4115-21 